\title{
LA REINSERCIÓN LABORAL: UN NUEVO RETO EN EL PACIENTE CON CÁNCER
}

\section{RETURNING TO WORK: A NEW CHALLENGE TO CANCER PATIENT}

\author{
Raquel Molina y Jaime Feliv ${ }^{2}$ \\ 1 Servicio de ESI/Oncología. Hospital Universitario Príncipe de Asturias. Universidad de Alcalá. Madrid. \\ 2 Servicio de Oncología. Hospital Universitario La Paz. Universidad Autónoma de Madrid.
}

Resumen

Los avances en el diagnóstico y tratamiento de los tumores malignos han dado relevancia a temas psicosociales como la reinserción laboral en los supervivientes a un cáncer. En esta revisión se analizan los trabajos más importantes acerca de los cambios laborales que tienen estos pacientes y su relación con distintas variables de la enfermedad y de su entorno. Es necesario promover la investigación en este campo y establecer los mecanismos necesarios que faciliten el proceso.

Palabras clave: Reinserción laboral; supervivientes de cáncer; trabajar después del cáncer.

\section{Abstract}

With improvements in diagnosis and treatment, returning to work after cancer is an important issue in survivors. This is a review of the studies have investigated the frequency of changes in work situation due to cancer and analyzed the association with different variables but more information and support is needed to help patients with cancer to manage impairments in workplace.

Key words: Return to work; cancer survivors; work after cancer.

der incorporarse a un empleo supone para el superviviente un parámetro importante ya que, entre otras cosas, implica una estabilidad económica y una "normalización" de su vida. Algunos pacientes con cáncer son capaces de continuar trabajando mientras están siendo tratados y muchos otros vuelven a trabajar después del tratamiento. Como ocurre en otros trabajadores, los enfermos con neoplasias utilizan el empleo como recurso social y económico pero, además, tiene un significado especial porque les da oportunidad de verificarse a ellos mismos y les permite ganar confianza sobre su salud y su estado social ${ }^{(2-4)}$.

\section{Correspondencia:}


La reincorporación al trabajo es una de las metas obligadas del proceso de rehabilitación de cualquier enfermedad y, por lo tanto, también para el cáncer. Requiere un acercamiento multidisciplinario coordinado para lograr preservar o mejorar el funcionamiento y maximizar la independencia de estos pacientes.

\section{DESARROLLO}

En los últimos años ha comenzado a haber un número creciente de estudios acerca de las consecuencias psicosociales del cáncer, y, dentro de ellos, relacionados con el impacto que esta enfermedad tiene en los aspectos laborales de los supervivientes. En muchos países del norte de Europa se están dedicando esfuerzos específicos a través de los institutos de Salud Laboral y de los terapeutas ocupacionales, pero en nuestro país es una asignatura pendiente.

En cuanto al coste que supone la implantación de estas medidas, Taylor et al. demostraron que el coste de la rehabilitación que requiere un paciente oncológico es inferior a la que supone la de un paciente con diabetes, tuberculosis, un trastorno mental, un problema ortopédico o con una patología cardiaca(5). En un estudio llevado a cabo en EEUU se demostró que los supervivientes de una neoplasia son tan productivos como otros trabajadores y que no tienen una tasa de absentismo laboral mayor que el resto ${ }^{(6)}$. Uno de los principales conflictos en lo referente a los empleadores es que cuestionan los problemas que pueden tener con cada persona en el trabajo a priori. En el caso del cáncer son similares a los de otras patologías crónicas ${ }^{(7,8)}$.

La tasa media de reinserción laboral en la mayoría de los estudios más significativos realizados en EEUU y algunos países europeos, oscila entre el 30 y el $92 \%$, con una media del $62 \%{ }^{(9)}$. En los trabajos más recientes y significativos que analizan este factor la tasa es del 35\%(10), 50\%(11) y $67 \%{ }^{(12)}$. Por tanto, una buena parte de los supervivientes son capaces de continuar trabajando o volver al trabajo sin limitaciones resultantes de su diagnóstico o de su tratamiento ${ }^{(13)}$. Ya en uno de los primeros estudios sobre el tema, realizado en los empleados de la Compañía Aseguradora del Metropolitano y la Compañía de Teléfonos y Telégrafos americana se comunicó que el desempeño del trabajo, el absentismo y el recambio laboral eran comparables entre las personas con y sin cáncer. No se encontró ningún impacto de la enfermedad neoplásica en la mayoría de los criterios relacionados directamente con el trabajo ${ }^{(14)}$.

A pesar de todo, hay un sentimiento persistente desde la perspectiva de los empleados de que las cosas no son iguales. Una trilogía de estudios sobre discriminación laboral fueron emprendidos por Feldman y cols identificaban varias formas de discriminación en el lugar de trabajo y problemas de adaptación como consecuencia de las secuelas de esta enferme$\operatorname{dad}^{(15-17)}$.

En el estudio finlandés de TaskilaAbrandt et al. se objetivó que los supervivientes de una neoplasia tenían un 9\% menos de probabilidades de volver a trabajar con respecto al resto de la población. Se debía, principalmente, a una mayor tasa de jubilaciones precoces ${ }^{(11)}$. En otro estudio realizado en el año 2000, se encontró que, con respecto a la población general, el $16,8 \%$ de los supervivientes (comparado con el $5 \%$ de controles de población sin una neoplasia) no eran capaces de trabajar a causa de problemas físicos, mentales o emocionales. Además, el $7,4 \%$ de los que podían trabajar estaban limitados en la cantidad o en el tipo de actividad laboral que podían desempeñar mientras que esto ocurrió en el 3,2\% de los controles ${ }^{(18)}$. 
En un estudio reciente realizado en supervivientes de cáncer en Pensilvania se objetivó que una de cada 7 de estas personas no recibían ninguna ayuda en los aspectos laborales ${ }^{(19)}$.

Hay dos metanálisis recientes y una revisión sistemática de la literatura que abordan los estudios centrados en la reinserción laboral de los pacientes con cáncer en general. El primero de ellos fue publicado por Steiner et al. ${ }^{(20)}$ en el año 2004 y es un metanálisis de distintas bases de datos desde 1966 al 2003. Sólo se encontraron 4 estudios que cumplieran varios de los requerimientos metodológicos que establecieron los autores; se trataba de trabajos con más de 235 pacientes, que abordaban distintos tipos de tumores, que estaban cercanos al diagnóstico de la enfermedad y que utilizaban para la selección, pacientes consecutivos ante la falta de registro de tumores. Se obtuvo un porcentaje de vuelta al empleo del $50-75 \%$ y los factores que más influían en ello era la ausencia de secuelas, sobre todo astenia, y el realizar un trabajo predominantemente no físico.

El segundo metanálisis relevante es del año 2009, publicado por De Boer et al.(21). Está centrado en aquellos estudios realizados con un grupo control entre los años 1966 y 2008. Incluyó 26 estudios, de los cuales 22 fueron publicados a partir del año 2002. La mayoría eran trabajos centrados en el carcinoma de mama y en tumores hematológicos. El resultado fue que los supervivientes de cáncer tenían 1,37 más riesgo de desempleo que los controles, aunque las tasas eran similares en los tumores hematológicos, germinales y en el cáncer de próstata.

Más recientemente, se ha publicado una revisión sistemática de la literatura entre los años 2000 y 2009 basada en 64 estudios originales centrados en la vuelta al trabajo de los enfermos con cáncer. El porcentaje fue del $63,5 \%$ con una ausencia media de 121 días. Los factores relaciona- dos con una mejor reinserción fue la flexibilidad horaria en el lugar de trabajo, el tener una menor edad, el sexo masculino, la ausencia de secuelas y el seguimiento médico y la rehabilitación laboral a lo largo de todo el proceso de la enfermedad ${ }^{(22)}$. En relación con estos dos últimos puntos, se han publicado ya distintos trabajos en los que se refuerza la idea de que el llevar a cabo una terapia ocupacional a la vez que el tratamiento específico del tumor, mejora la reinserción laboral (23-25).

¿Y qué ocurre en nuestro país? En España hay pocos trabajos publicados sobre este tema. El trabajo más pionero fue publicado en el año 95 por Martos et al.(26). Se trataba de una serie de 45 pacientes laringuectomizados por un carcinoma y a los dos años de la remisión completa de la enfermedad. Se obtuvo una tasa muy baja de reinserción $(27 \%)$ y estaba en relación con la adquisición de erigmofonación y el ser autónomo ${ }^{(26)}$.

En el año 2000, nuestro grupo realizó un estudio piloto en el Hospital La Paz con 95 pacientes diagnosticados de diferentes neoplasias (predominaban los tumores gastrointestinales y los linfomas) tras adyuvancia o una primera línea de tratamiento. Se obtuvo una tasa de reinserción del 70\% y los factores que influían de forma negativa en el proceso fueron el ser asalariado y la presencia de secuelas ${ }^{(27)}$.

Basándonos en este estudio piloto, se realizó un trabajo con 347 pacientes que ha permitido, por primera vez, hacerse una idea de la situación general de la actividad laboral de los enfermos con una neoplasia en nuestro país y la elaboración de un "Índice Pronóstico de Actividad Laboral". Asimismo, estudió la influencia de distintas variables en dos momentos claves de la enfermedad como es al inicio del tratamiento específico antineoplásico y al finalizar éste, y recogió las percepciones subjetivas de los propios encuestados sobre distintos aspectos de su situación labo- 
ral y del entorno en el que se desarrollaba su trabajo. La mayor parte de los pacientes $(85 \%)$ estuvieron inactivos tras comenzar el tratamiento del tumor. Este hecho se relacionó en mayor medida con el sexo masculino, una edad mayor de 45 años, el tener un régimen laboral asalariado y la utilización de quimioterapia como modalidad terapéutica.

Una vez terminado el tratamiento, el $41 \%$ de los entrevistados estuvo de baja laboral. En este caso, se objetivó la influencia de una edad mayor de 45 años, el tener estudios no universitarios, el realizar un trabajo predominantemente físico, la presencia de otras enfermedades concomitantes, el no conseguir una remisión completa de la enfermedad y las secuelas de la propia neoplasia o del tratamiento recibido.

Se identificaron como factores predictivos independientes para la actividad laboral al comienzo del tratamiento una edad menor de 45 años, el no recibir quimioterapia y el régimen laboral autónomo. Sin embargo, tras la finalización del tratamiento fueron significativos una edad menor de 45 años, el tener una remisión completa de la enfermedad tumoral y la ausencia de secuelas derivadas de la propia neoplasia o de la modalidad terapéutica utilizada. En cuanto al entorno laboral, la mayoría de los pacientes habían contado a sus compañeros y/o jefes que habían tenido o tenían un tumor maligno y no habían percibido cambios en la actitud de éstos. En la minoría en que sí se observaron, se recogieron predominantemente, cambios positivos. La mayoría de los entrevistados opinaron que la incapacidad laboral era debida a la enfermedad tumoral o a las secuelas derivadas de ella. No pensaban que la realización de cambios en su lugar de trabajo facilitarían su vuelta a él. Tampoco ocultarían su historial médico al buscar un nuevo empleo ${ }^{(28)}$.

\section{CONCLUSIONES}

Al igual que otros muchos aspectos físicos y psicológicos del cáncer, la rehabilitación laboral es un aspecto en el que hay que incidir desde etapas tempranas de la enfermedad ya que, como se ha visto, supone un importante índice psicosocial de calidad de vida para estos pacientes.

Por tanto, es fundamental seguir investigando en este campo y que sea en nuestro medio ya que las comparaciones entre distintos países están muy influenciadas por los distintos regímenes estatales de cada uno de ellos. Sería muy recomendable además, potenciar una educación pública y profesional para la desaparición de los Ilamados "mitos" del cáncer junto con el desarrollo de programas específicos.

La búsqueda de factores predictivos de la vuelta al trabajo permite poder identificar aquellos que pueden ser controlables. Algunos importantes predictores de la reinserción laboral encontrados como la edad al diagnóstico, la localización o el estadio tumoral y la situación actual de la enfermedad, no se pueden modificar por intervenciones específicas. En cambio, son candidatos para una modificación y una mejora otros como las secuelas producidas por la neoplasia en sí o por el tratamiento específico recibido, las creencias o los mitos que se desarrollan en el entorno laboral, las barreras físicas que un enfermo pueda encontrar en él o, simplemente, el propio pensamiento del paciente de que el tumor le puede perjudicar en su vida laboral. En este sentido, hay que insistir en el desarrollo de tratamientos que, manteniendo o mejorando la eficacia, produzcan menos secuelas y en la detección precoz de aquellas reversibles para instaurar una terapéutica apropiada y que no lleguen a ser invalidantes en un futuro más o menos próximo. Aunque pueda que no exista una repercusión actual en la reincorporación laboral, a largo plazo pueden conllevar un 
peor estado de salud que influya en una jubilación precoz. Esto último se plantea en un estudio realizado recientemente en Noruega en el que no se detectaron diferencias en la actividad laboral en una muestra de pacientes con una historia de enfermedad tumoral frente a población sana, pero en los que se encontraron diferencias significativas en la presencia de una peor situación física y mental de los primeros ${ }^{(29)}$.

El desarrollo de equipos multidisciplinarios de rehabilitación compuestos por médicos, enfermeras, fisioterapeutas, terapeutas ocupacionales, especialistas en foniatría y asistentes sociales, que tengan en cuenta los problemas individuales de cada paciente es otro de los puntos a proponer. Los pacientes entrevistados en el trabajo de Maunsell se quejaron de la falta de atención de los profesionales médicos en el tema de los problemas laborales ${ }^{(30)}$. Por otro lado, en un estudio llevado a cabo por Verbeek et al., se encontró como factor predictivo en la vuelta al trabajo después de haber tenido un tumor maligno, la continuidad de cuidados por un terapeuta ocupacional ${ }^{(23)}$. A esta misma conclusión se había llegado antes en pacientes con dolor de columna(31).

Se debe establecer una cierta flexibilidad en las consultas tanto para los tratamientos como para las revisiones, con el fin de que su asistencia interfiera lo menos posible con las obligaciones laborales.

Además, es necesaria una educación pública y profesional para la desaparición de los llamados "mitos" del cáncer y soluciones legales para la igualdad de oportunidades, en las que se valore a la persona por sus capacidades y no por sus antecedentes personales. En EEUU ya existen cuatro leyes federales que favorecen la protección de los pacientes con enfermedades crónicas entre los que están, específicamente, los supervivientes con cáncer ${ }^{(13)}$ : Americans with Disabilities Act
(ADA), Federal Rehabilitation Act, Family and Medical Leave Act (FMLA) y Employee Retirement and Income Security Act (ERISA). Una vez promulgada la base legal se deberían destinar recursos locales y estatales para la reintegración a través del desarrollo de programas específicos.

En las empresas y otros lugares de trabajo son necesarios esfuerzos para el cumplimiento de los derechos establecidos y una serie de acomodaciones especiales que faciliten la reinserción con asistencia en los casos de incapacidad específicos de esta enfermedad.

Por último, la creación de asociaciones de supervivientes permite un apoyo independiente y eficaz en esta tarea.

Tenemos una ardua tarea para conseguir todos estos objetivos en nuestro medio.

\section{REFERENCIAS BIBLIOGRÁFICAS}

1. Mullan F. Season of survival: Reflections of a physician with cancer. New Engl J Med 1985; 313:270-3.

2. Gambosi J. Recovering from cancer: a nursing intervention programme. Oncol Nurs Forum 1990; 17(2): 215-219.

3. García C, Fernández Y, Gómez J, Salinas P. Los supervivientes del cáncer. En: González Barón M, Ordóñez A, Feliu J, Zamora P, Espinosa E, editores. Tratado de medicina paliativa y tratamiento de soporte en el enfermo con cáncer. Madrid: Panamericana, 1995. p. 1344-58.

4. Taskila T, Lindbohm ML. Factors affecting cancer survivors'employment and work ability. Acta Oncol 2007; 46(4):446-51. Doi: 10.1080/02841860701355048.

5. Taylor CM. The rehabilitation of persons with cancer: is the best we can do? J Rehabil 1984; 50: 60-71.

6. National Institute of Health. Facing forward. A guide for cancer survivors. Washington DC, US. Department of Health and Human Services. NIH publication No. 1990; 902424. 
7. Bradley CJ. The need for online information on the economic consequences of cancer diagnosis, treatment and survivorship. J Med Internet Res 2005; 7(3): e29. Doi: 10.2196/jmir.7.3.e29.

8. McAleer CA, Kluge CA: Why cancer rehabilitation? Rehabil Couns Bull 1978; 21: 208-15.

9. Chirikos TN, Russell-Jacobs A, Cantor AB. Indirect economic effects of longterm breast cancer survival. Cancer Pract 2002; 10: 248-55. Doi: 10.1046/j.15235394.2002.105004.x.

10. Spelten ER, Sprangers MA, Verbeek JH. Factors reported to influence the return to work of cancer survivors: A literature review. Psychooncology 2002; 11: 124-31. Doi: 10.1016/S0959-8049(03)00364-2.

11. Taskila-Abrandt T, Pukkala E, Martikainen R, Karjalainen A, Hietanen P: Employment status of finnish cancer patients in 1997. Psychooncology 2005; 14: 221-6. Doi: 10.1002/pon.838.

12. Salander $P$, Bergenheim AT, Henriksson R: How was life after treatment for malignant brain tumors? Soc Sci Med 2000; 51: 589-98.

13. Schultz PBM, Stava C, Sellin R. Cancer Survivors. Work related issues. Am J Occup Health Nurs 2002; 50: 220-6.

14. Hoffman B. Cancer survivors al work: a generation of progress. CA Cancer J Clin 2005; 55: 271-80. Doi: 10.1002/ cncr.24301.

15. Feldman F. The return to work: A question of workability. In: American Cancer Society. Proceedings of the Workshop on Employment, Insurance and the Patient with cancer. New Orleans 1987; 27-35.

16. Wheatley GM, Cunnick WR, Wright BP, Van Keuren D. The employment of persons with a history of treatment for cancer. Cancer 1974; 33: 441-5.

17. Feldman FL. Work and cancer health histories: Study of the experiences of recovered patients. San Francisco. American Cancer Society 1976.
18. Feldman F: Work and cancer health histories: A study of the experiences of recovered blue-collar workers. San Francisco. American Cancer Society. California Division Inc 1978; 340-341.

19. Barg FK, Cronholm PF, Straton JB, Keddem S, Knott K, Grater J, et al. Unmet psychosocial needs of Pennsylvanians with cancer: 1986-2005. Cancer 2007; 110: 631-9. Doi: 10.1002/cncr.22820.

20. Steiner JF, Cavender TA, Main DS, Bradley CJ. Assessing the impact of cancer on work outcomes. Cancer 2004; 15: 1703-11. Doi: 10.1002/cncr.20564.

21. de Boer AG, Taskila T, Ojajarvi A, van Dijk FJ, Verbeek JA. Cancer survivors and unemployed: a meta-analysis and metaregression. JAMA 2009; 301(7): 753-62. Doi: 10.1001/jama.2009.187.

22. Mehnert A. Employmnet and work-related issues in cancer survivors. Crit Rev Oncol Hematol 2010; 28: 651-6. Doi: 10.1016/j. critrevonc.2010.01.004.

23. Verbeek J, Spelten E, Kammeijer M, Sprangers M. Return to work of cancer survivors: A prospective cohort study into the quality of rehabilitation by occupational physicians. Occup Environ Med 2003; 60: 352-7. Doi: 10.1136/oem.60.5.352.

24. Frazier LM, Miller VA, Miller BE, Horbert DV, Delmore JE, Ahlers-Schimdt CR. Cancer-related task involving employment: opportunities for clinical assistance. J Support Oncol 2009; 7(6): 229-36. Doi: 10.1177/1049732310361466.

25. Kennedy F. Returning to work following cancer: A qualitative exploratory study into the experience of returning to work following cancer. Eur J Cancer Care 2007; 16:17-25. Doi: 10.1111/j.1365-2354.2007.00729.x.

26. Martos C, Sánchez I, Martínez R, García C, Álvarez M, Larrubia I, et al. Reinserción sociolaboral tras laringuectomía total. Rehabilitación 1995; 29(2): 85-91.

27. Jiménez A, Feliu J, Molina R, Ríos E, Zamora P, González Barón M, Reinserción 
laboral del paciente neoplásico. Oncología 2000; 23(7): 318-26.

28. Molina R, Feliu J, Villalba A, San José B, Jiménez AM, Espinosa $E$, et al. Employment in a cohort of cancer patients in Spain. Clin Transl Oncol 2008; 10:826-30. Doi: 10.1007/s12094-008-0296-4.

29. Gudbergsson SB, Fossa SD, Borgeraas E, Dahl AA. A comparative study of living conditions in cancer patients who have returned to work after curative treatment.
Support Care Cancer 2006; 14(10): 10209. Doi: 10.1007/s00520-006-0042-9.

30. Maunsell E, Brisson C, Dubois L, Lauzier S, FraserA. Work problems after breast cancer: An exploratory qualitative study. Psychooncology 1999; 8: 467-473. Doi: 10.1002/(SICI)10991611 ( $199911 / 12) 8: 6<467:$ : AID PON400>3.0.CO;2-P.

31. Waddel G, Burton AK. Occupational health guidelines for the management of low back pain at work: Evidence review. Occup Med 2001; 51: 124-135. 
